\title{
IS PORCINE KOBUVIRUS 1 A TYPICAL DIARRHOEIC PATHOGEN OF PIGLETS?
}

\author{
McFall, H., Vilček, Š. \\ Department of Epizootology and Parasitology \\ University of Veterinary Medicine and Pharmacy, Komenského 73, 04181 Kosice, \\ Slovakia \\ stefan.vilcek@uvlf.ak
}

\section{ABSTRACT}

The objective of this study was to show if porcine kobuvirus 1 (PKV-1) participates in the development of diarrhoea in piglets. The experiments were focused on comparing the occurrence of PKV-1 with the occurrence of rotavirus A (RVA) infection in suckling pigs on Slovak pig farms. A total of 91 rectal swabs of piglets (age $<28$ days) were collected from 8 pig farms. RT-PCR was employed to detect PKV-1 through amplification of the $495 \mathrm{bp}$ fragment of the 3D gene using primers KoVF/ KoVR, and RVA was detected through amplification of the $309 \mathrm{bp}$ fragment of the VP6 gene using primers rot3 and rot5. As expected, the detection of RVA in diarrhoeic piglets was $56.8 \%(P<0.01)$, while only $14.8 \%$ in healthy animals. These results confirm that RVA is one of the main causes of diarrhoea in young piglets. Comparatively, PKV-1 was detected in approximately equal numbers in the same group of both healthy and diarrhoeic pigs, with $\mathbf{7 4 . 1} \%$ in healthy animals and $81.1 \%$ in diarrhoeic animals, which was not statistically significant $(P<0.05)$. The level of co-infection of both viruses was
$11.1 \%$ in healthy animals. A portion of $48.6 \%(\mathrm{P}<0.01)$ of diarrhoeic animals were found with RVA and PKV-1 coinfections. The results of this study indicate that while RVA is an enteric virus, PKV-1 cannot confidently be confirmed as an enteric pathogen.

Key words: diarrhoea; piglet; porcine kobuvirus; rotavirus

\section{INTRODUCTION}

One of the major health problems in the swine industry is the high frequency of diarrhoea in piglets, which is one of the main causes of mortality and morbidity in neonatal pigs. The aetiology of enteric disease is diverse, including viral, bacterial and protozoal pathogens, but viruses are the predominant factor. In 2008, a novel virus was detected in faecal samples from clinically healthy pigs in Hungary [21]. This virus belongs to the family Picornaviridae and demonstrates similarity to members of the genus Kobuvirus; Aichivirus A (previously known as human aichi virus) and 
Aichivirus $B$ (formerly bovine kobuvirus). The virus was named porcine kobuvirus $1(\mathrm{PKV}-1)$, which is also referred to as Aichivirus $C$.

The kobuvirus genome ranges from 8.2 to $8.4 \mathrm{~kb}$ in size with a viral protein genome (VPg) linked to the 5'-untranslated region (UTR) and a poly (A) tail at the 3'-UTR [12]. The polyprotein precursor is further processed to generate a leader (L) protein, three structural proteins (VP0, VP3 and VP1), and seven non-structural proteins (2A, 2B, 2C, 3A, 3B, 3C and 3D) [23]. The RNA-dependent RNA polymerase (3D) is the most conserved region among kobuviruses [22].

PKV-1 has been detected in diarrhoeic pigs either in individual infections or in mixed infections with other established diarrhoeic viruses. In more recent years, the virus has been detected worldwide, with studies demonstrating PKV-1 in Asia [10, 14, 16, 17, 28,], Europe [7, 13, 21, 29], North America [25], South America [2] and East Africa [1]. In the scientific literature, PKV-1 is traditionally described as an enteric virus, however, its pathogenesis and contribution to the development of enteric disease remain poorly understood.

Rotaviruses were first described in pigs in 1976, and they are known enteric pathogens which cause gastroenteritis in young children and animals [27]. Classification of members of the Rotavirus genus is based on the serological reactivity and genetic variability of VP6, and at present there are 8 different groups which are differentiated alphabetically from A-H (RVA-RVH) [18]. The RVA group is antigenically the most diverse species amongst the Rotavirus genus, and they are also the most important due to their high prevalence and pathogenicity in both humans and animals.

The goal of this study was to determine the prevalence of porcine kobuvirus 1 in both healthy and diarrhoeic piglets in Slovakia, and to determine the relationship between kobuvirus and rotavirus A in their ability to cause diarrhoea. This information will contribute to the present knowledge available on porcine kobuvirus 1 , and ultimately aid the understanding of its clinical impact on the swine industry.

\section{MATERIALS AND METHODS}

\section{Samples}

Rectal swabs were collected from 8 farms across Eastern and Western Slovakia, using transport swab applica- tors (Sarstedt AG\&Co, Germany). The samples were collected from 91 suckling piglets before they were of weaning age ( $<28$ days) which were divided into clinically healthy $(\mathrm{n}=54)$ and diarrhoeic $(\mathrm{n}=37)$ groups

\section{RNA isolation}

Elution of the rectal swabs was achieved by adding $1 \mathrm{ml}$ of 0.01 mol. $1^{-1}$ PBS (Merck Millipore Corp., USA) and then allowing the samples to incubate for 30 minutes. The samples were then vortexed for 3 minutes at 2000 rev. $\mathrm{min}^{-1}$ and then centrifuged for 5 minutes at $14,000 \times \mathrm{g}$. Then $200 \mu \mathrm{l}$ of the sample was added to $700 \mu \mathrm{l}$ of TRIzol Reagent (Life Technologies, USA), followed by gentle hand-mixing and a $5 \mathrm{~min}$ incubation. $200 \mu \mathrm{l}$ of chloroform was added to each sample to better separate the solution into water and organic phases. $500 \mu \mathrm{l}$ of the top aqueous layer was removed and then $500 \mu \mathrm{l}$ isopropyl alcohol was added to each test tube. The precipitated nucleic acid was washed with $75 \%$ alcohol.

\section{cDNA synthesis}

The synthesis of cDNA was carried out using reverse transcrip2196tase and random hexamers as described by Jackova et 98al. [13].

\section{Detection of rotavirus $A$ and porcine kobuvirus 1 using single RT-PCR}

Amplification of a $309 \mathrm{bp}$ fragment of the VP6 gene was performed to detect the RVA genome, by using primers rot3 and rot5 [9]. For the detection of PKV-1 with PCR, the same cDNA, as prepared for RVA, was used. The single RTPCR for PKV-1 was based on the amplification of a 495 bp fragment of the 3D gene using primers KoVF/KoVR [28]. Details of both PCR methods were described elsewhere [13].

\section{Gel electrophoresis}

The size of the PCR products was checked by electrophoresis in $2 \%$ agarose gel. The DNA Ladder 100 bp marker (AppliChem, GmbH, Germany) was used to aid approximation of the size of the DNA fragments.

\section{Statistical analysis}

Statistical analysis was performed using the software GraphPad Prism 5 for Windows (GraphPad Software, USA). The data was analysed using the chi-square $(\chi 2)$ test, with 
confidence limits of $95 \%, \mathrm{P}<0.05$ (statistically significant) or $99 \%, \mathrm{P}<0.01$ (highly statistically significant).

\section{RESULTS}

Prevalence of rotavirus A in clinically healthy and diarrhoeic pigs

An electrophoresis image for RVA detection is displayed in Fig. 1A, based on RT-PCR amplification of the 309 bp DNA fragment. Results on the detection of RVA in healthy and diarrhoeic piglets are presented in Fig. 2. The total percentage of positive suckling pigs for rotavirus $\mathrm{A}$ was $31.9 \%$ (29/91). Only 8 healthy animals were positive, which produces a positivity rate of $14.8 \% .21$ of the $37 \mathrm{di}$ arrhoeic suckling pigs tested positive for rotavirus, giving a $56.8 \%$ figure for positive diarrhoeic animals. The chisquare $\left(\chi^{2}\right)$ test showed that there was a highly statistically significant correlation (level of $99 \%$ ) between RVA and diarrhoea $(\chi 2=17.7887 ; \mathrm{P}=0.000025)$.
Prevalence of porcine kobuvirus 1 in clinically healthy and diarrhoeic pigs

An electrophoresis image for PKV-1 detection is displayed in Fig. 1B. Clinical samples positive for PKV-1 were represented with a 495 bp electrophoretic band. The summary of results on the detection of PKV-1 RNA in the clinical samples is presented in Fig. 2. Of the 91 suckling pigs, 70 tested positive for $\mathrm{PKV}-1$, which produces a positive percentage of $76.9 \%$. Forty of the healthy 54 suckling pigs were PKV-1 positive, representing a $74.1 \%$ positivity rate. Diarrhoeic suckling pigs showed a higher positive percentage of $81.1 \%$, with 30 of the 37 diarrhoeic pigs testing positive. Despite observation of some differences between healthy and diarrhoeic animals, there was no statistically significant correlation at the level of $95 \%(\mathrm{P}<0.05)$ between PKV-1 and diarrhoea $(\chi 2=0.6072 ; \mathrm{P}=0.4358)$.

\section{Levels of co-infection of rotavirus $A$ and porcine} kobuvirus 1 in clinically healthy and diarrhoeic pigs

Both PKV-1 and RVA were detected together in 24 pigs, with a positivity rate of $26.4 \%$. Healthy pigs had a positivity rate of $11.1 \%$ (6 positive; 48 negative), whereas a higher rate of $48.6 \%$ was detected in diarrhoeic pigs (18 positive;

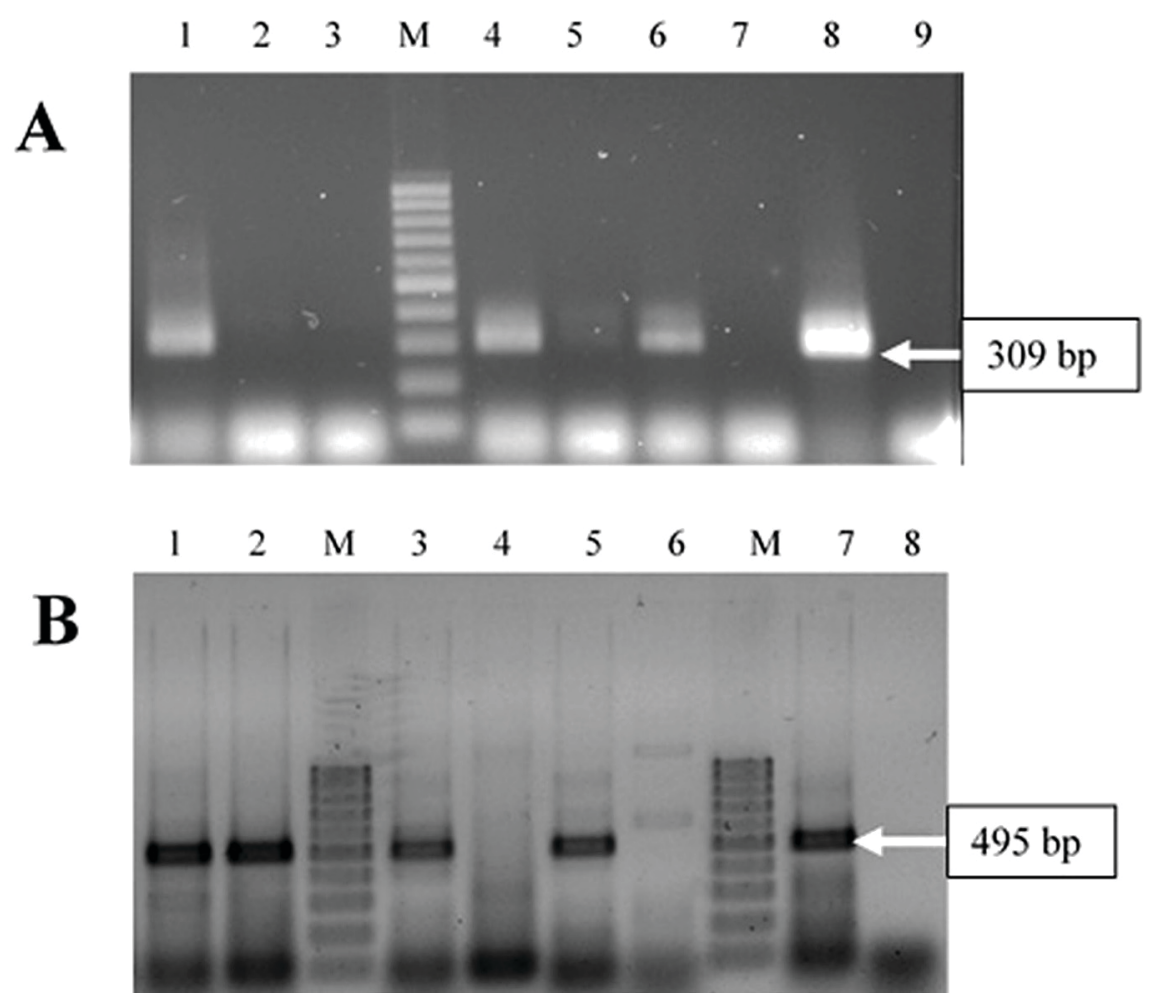

Fig. 1. Gel electrophoresis of PCR products. A - Samples 1, 4, 6 and 8 are positive for rotavirus A $\mathrm{B}-$ samples $1,2,3,5$ and 7 are positive for porcine kobuvirus 1 


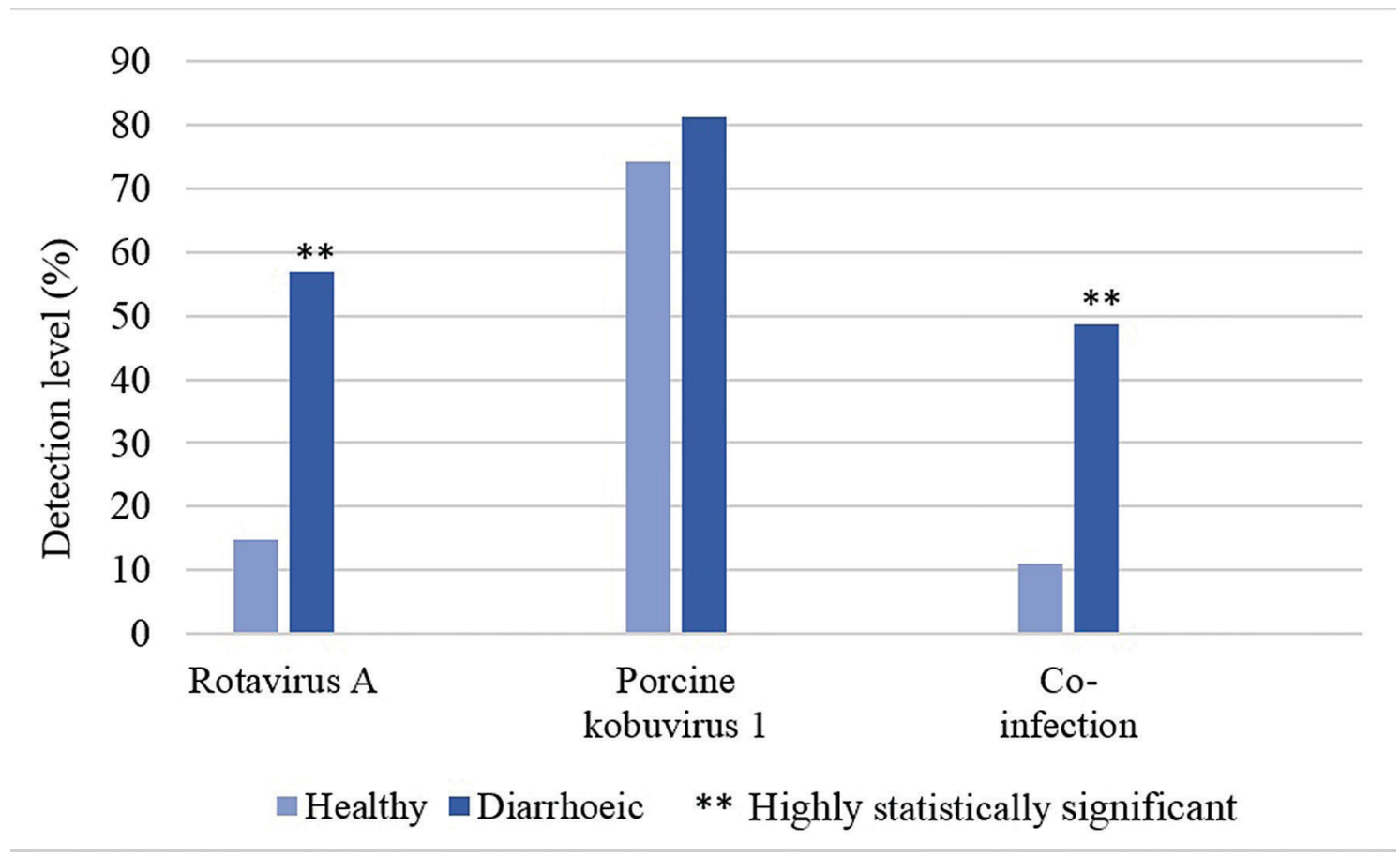

Fig. 2. Comparison within the same group of piglets for the levels of detection of rotavirus A, porcine kobuvirus 1 and co-infection

19 negative). There was a highly statistically significant correlation (level of $99 \%, \mathrm{P}<0.01$ ) between co-infection and diarrhoea $(\chi 2=15.9324 ; \mathrm{P}=0.000066)$. All results on $\mathrm{co}-$ infection study are summarized in Fig. 2.

\section{DISCUSSION}

It is important to determine the prevalence of PKV-1 and RVA in pigs within a region, in order to contribute to the available knowledge on the global prevalence of these viruses and their role in porcine diarrhoea. The results from this study indicated that there was a highly statistically significant correlation between RVA infection and the occurrence of diarrhoea. Comparatively, no statistically significant difference was determined between PKV-1 infection and diarrhoea.

The overall prevalence of RVA in piglets was $31.9 \%$. Similar levels of incidence were also detected in Slovenia and Spain, with $20.0 \%$ and $16.7 \%$, respectively [19]. In contrast, other European countries showed a lower incidence of RVA, such as $4.2 \%$ in Hungary, and $10.1 \%$ in Denmark [19].
RVA was detected in $56.8 \%$ of diarrhoeic piglets but only in $14.8 \%$ of clinically healthy piglets. This result was relatively anticipated, as RVA has been proven in scientific literature to be a predominant enteric pathogen and therefore a cause of diarrhoea. Publications on the association between RVA and diarrhoea are long-established, since its discovery in pigs in 1976 [27]. It is believed that group A rotaviruses are responsible for $53 \%$ of pre-weaning and 44 $\%$ of post-weaning diarrhoea in pigs [11]. Specifically regarding RVA, it is reported to account for $89 \%$ for all rotavirus diarrhoea in commercial pig populations [26]. The prevalence of diarrhoea in the sampled age group of piglets also corresponds with the scientific literature, as RVA infections tend to have the greatest implications on animals less than 28 days old $[15,24]$.

In total, a $76.9 \%$ positivity rate was determined for PKV-1 infection in pigs originating from Slovakia. This figure of prevalence within Slovakia concurs with the European levels detected by Zhou et al. [29] in a study across 5 European countries (Austria, Germany, Hungary, Spain and Sweden), with an overall prevalence of $56.7 \%$. When comparing the levels of PKV-1 in healthy and diarrhoeic pigs, the present study detected nearly similar levels 
in both clinical categories, with $74.1 \%$ in healthy animals and $81.1 \%$ in diarrhoeic animals. Zh ou et al. [29] detected similar levels, with an average finding of $54.5 \%$ in healthy pigs in the 5 European countries, and $58.2 \%$ detected in diarrhoeic pigs.

PKV-1 was incidentally detected at a level of $13.3 \%$ in clinically healthy animals in Hungary when searching for astroviruses [21]. Since then, many studies have aimed to compare the level of detection in healthy and diarrhoeic animals. The successive study was performed by $\mathrm{Yu}$ et al. [28], in which they discovered a $30.1 \%$ prevalence rate amongst clinically healthy pigs in China. More recent studies, such as that performed by Di Bartolo et al. [6] on Italian farmed pigs, subsequently confirmed the results of previous studies, with PKV-1 detection levels of $57.5 \%$ in healthy pigs and $49.7 \%$ in diarrhoeic pigs.

However, other studies have demonstrated higher levels of PKV-1 in diarrhoeic animals, which suggests a potential link to causing enteric disease. Kh a m rin et al. [16] identified a $99 \%$ positivity rate for $\mathrm{PKV}-1$ in diarrhoeic pigs in Thailand, however this study did not provide a comparison with the levels of PKV-1 in asymptomatic pigs. A study in Korea by Park et al. [20] stated a statistically significant correlation between the occurrence of diarrhoea and PKV-1, however, only $3.57 \%$ of the diarrhoeic pigs tested for PKV-1 alone, thereby the possibility of another enteric pathogen causing diarrhoea cannot be excluded.

If kobuvirus infection was responsible for causing diarrhoea, we would expect to see a statistically significant correlation between diarrhoea and PKV-1, though this was not confirmed in the present study. Similar difficulty in establishing significance is also true for other emerging viruses, including porcine sapovirus, porcine enterovirus $\mathrm{G}$ and porcine astrovirus, as they are also detected in healthy and diarrhoeic pigs without known clinical significance $[3,4,5,8]$.

The levels of co-infection were also investigated in this study. A total of 24 out of the 91 sampled piglets tested positive for both RVA and PKV-1, giving a percentage of $26.4 \%$. When comparing the prevalence of co-infection between healthy and diarrhoeic animals, there was a highly statistically significant relationship between co-infection and diarrhoea. Only $11.1 \%$ of healthy animals were positive for both viruses, in comparison to $48.6 \%$ of diarrhoeic animals. Interpreting the individual factors of co-infection is a challenging task, as it is difficult to attribute the occurrence of diarrhoea with a specific aetiological agent.

\section{CONCLUSIONS}

The results of this study clearly confirmed that while RVA is a causative agent of diarrhoea in piglets, PKV-1 cannot confidently be confirmed as a typical diarrhoeic pathogen. Although PKV-1 has a high prevalence in piglets in Slovakia, they survive with absence of significant disease. Further investigation is required to determine the role of PKV-1 in the enteric system and such kind of research requires a more complex approach including the application of the next generation of sequencing techniques.

\section{ACKNOWLEDGEMENTS}

This study was prepared as a student scientific work which was a part of broader project under the supervision of Stefan Vilček. The study was supported by project INFEKTZOON (ITMS 26220120002) co-financed by EU.

\section{REFERENCES}

1. Amimo, J. O., Okoth, E., Junga, J. O., Ogara, W. O., Njahira, M. N., Wang, Q., et al., 2014: Molecular detection and genetic characterisation of kobuvirus and astroviruses in asymptomatic local pigs in East Africa. Arch. Virol., 159, 1313-1319.

2. Barry, A., Ribeiro, J., Alfieri, A., van der Poel, W., Alfieri, A., 2011: First detection of kobuvirus in farm animals in Brazil and the Netherlands. Infect. Genet. Evol., 11, 1811-1814.

3. Benedictis, P., Schultz-Cherry, S., Burnham, A., Cattoli, G., 2011: Astrovirus infections in humans and animals - molecular biology, genetic diversity, and interspecies transmissions. Infect. Genet. Evol., 11, 1529-1544.

4. Brnić, D., Prpić, J., Keros, T., Roić, B., Starešina, V., Jemeršić, L., 2013: Porcine astrovirus viremia and high genetic variability in pigs on large holdings in Croatia. Infect. Genet. Evol., 14, 258-264.

5. Collins, P. J., Martella, V., Buonavoglia, C., O’Shea, H., 2009: Detection and characterization of porcine sapoviruses from asymptomatic animals in Irish farms. Vet. Microbiol., 139, 176-182.

6. Di Bartolo, I., Angeloni, G., Tofani, S., Monini, M., Ruggeri, F. M., 2015: Infection of farmed pigs with porcine kobuviruses in Italy. Arch. Virol., 160, 1533-1536. 
7. Di Profio, F., Ceci, C., Di Felice, E., Marsilio, F., Di Martino,

B., 2013: Molecular detection of porcine kobuviruses in Italian swine. Res. Vet. Sci., 95, 782-785.

8. Dung, V.N., Anh, P.H., Cuong, V. N., Hoa, N. T., CarriqueMas, J., Hien, V. B., et al., 2014: Prevalence, genetic diversity and recombination of species $\mathrm{G}$ enteroviruses infecting pigs in Vietnam. J. Gen. Virol., 95, 549-556.

9. Elschner, M., Prudlo, J., Hotzel, H., Sachse, K. K., 2002: Nested Reverse Transcriptase-Polymerase Chain Reaction for the Detection of Group A Rotaviruses. J. Vet. Med. B., 49, 77-81.

10. Fan, S., Sun, H., Ying, Y., Gao, X., Wang, Z., Yu, Y., et al., 2013: Identification and characterization of porcine kobuvirus variant isolated from suckling piglet in Gansu province, China. Viruses, 5, 2548-2560.

11. Fitzgerald, G.R., Barker, T., Welter, M.W., Welter, C. J., 1988: Diarrhoea in young pigs: comparing the incidence of the five most common infectious agents. Vet. Med. Small Anim. Clin., 1, 80-86.

12. Flather, D., Semler, B. L., 2015: Picornaviruses and nuclear functions: Targeting a cellular compartment distinct from the replication site of a positive-strand RNA virus. Frontiers in Microbiology, 6, 1-17.

13. Jackova, A., Sliz, I., Mandelik, R., Salamunova, S., Novotny, J., Kolesarova, M., et al., 2017: Porcine kobuvirus 1 in healthy and diarrhoeic pigs: Genetic detection and characterisation of virus and co-infection with rotavirus A. Infect. Genet. Evol., $49,73-77$.

14. Jin, W. J., Yang, Z., Zhao, Z.P., Wang, W. Y., Yang, J., Qin, A. J., Yang, H.C., 2015: Genetic characterization of porcine kobuvirus variants identified from healthy piglets in China. Infect. Genet. Evol., 35, 89-95.

15. Katsuda, K., Kohmoto, M., Kawashima, K., Tsunemitsu, H., 2006: Frequency of enteropathogen detection in suckling and weaned pigs with diarrhoea in Japan. J. Vet. Diagn. Invest., $18,350-354$

16. Khamrin, P., Maneekarn, N., Kongkaew, A., Kongkaew, S., Okitsu, S., Ushijima, H., 2009: Porcine kobuvirus in piglets, Thailand. Emerg. Infect. Diseases, 15, 2075-2076.

17. Khamrin, P., Meneekarn, N., Hidaka, S., Kishikawa, S., Ushijima, K., Okitsu, S., Ushijima, H., 2010: Molecular detection of kobuvirus sequences in stool samples collected from healthy pigs in Japan. Infect. Genet. Evol., 10, 950-954.

18. Matthijnssens, J., Otto, P. H., Ciarlet, M., Desselberger, U., Van Ranst, M., Johne, R., 2012: VP6-sequence-based cutoff values as a criterion for rotavirus species demarcation. Arch. Virol., 157, 1177-1182.
19. Midgley, S. E., Bányai, K., Buesa, J., Halaihel, N., Hjulsager, C. K., Jakab, F., et al., 2012: Diversity and zoonotic potential of rotaviruses in swine and cattle across Europe. Vet. Microbiol., 156, 238-245.

20. Park, S., Kim, H., Moon, H., Song, D., Rho, S., Han, J., et al., 2010: Molecular detection of porcine kobuviruses in pigs in Korea and their association with diarrhoea. Arch. Virol., 155, 1803-1811.

21. Reuter, G., Boldizsar, A., Kiss, I., Pankovics, P., 2008: Candidate new species of kobuvirus in porcine hosts. Emerg. Infect. Diseases., 14, 1968-1970.

22. Reuter, G., Boldizsar, A., Pankovics, P., 2009: Complete nucleotide and amino acid sequences and genetic organization of porcine kobuvirus, a member of a new species in the genus Kobuvirus, family Picornaviridae. Arch. Virol., 154, 101-108.

23. Reuter, G., Boros, A., Pankovics, P., 2011: Kobuviruses - a comprehensive review. Rev. Med. Virol., 21, 32-41.

24. Svensmark, B., Askaa, J., Wolstrup, C., Nielsen, K., 1989: Epidemiological studies of piglet diarrhoea in intensively managed Danish sow herds. IV. Pathogenicity of porcine rotavirus. Acta Vet. Scand., 30, 71-76.

25. Verma, H., Mor, S., Abdel-Glil, M., Goyal, S., 2013: Identification and molecular characterization of porcine kobuvirus in U. S. swine. Virus Genes., 46, 551-553.

26. Will, L. A., Paul, P. S., Proescholdt, T. A., Aktar, S. N., Flaming, K.P., Janke, B.H., et al., 1994: Evaluation of rotavirus infection in diarrhoea in Iowa commercials pigs based on an epidemiologic study of a population represented by diagnostic laboratory cases. J. Vet. Diagn. Invest., 6, 416-422.

27. Woode, G. N., Bridger, J. C., Hall, G. A., Jones, J. M., Jackson, G., 1976: The isolation of reovirus-like agents (rota-viruses) from acute gastroenteritis of piglets. J. Med. Microbiol., 9, 203-209.

28. Yu, J., Jin, M., Zhang, Q., Li, H., Li, D., Xu, Z., et al., 2009: Candidate porcine kobuvirus, China. Emerg. Infect. Diseases, $15,823-825$.

29. Zhou, W., Ullman, K., Chowdry, V., Reining, M., Benveda, Z., Baule, C., et al., 2016: Molecular investigations on the prevalence and viral load of enteric viruses in pigs from five European countries. Vet. Microbiol., 182, 75-81.

Received May 10, 2017

Accepted May 31, 2017 